\title{
HPLC Characterization and Assessment of Antioxidant Status of Vetiveria Zizanioides Roots
}

\author{
Punithavathi Manogaran, Suriyavathana Muthukrishnan, Kavitha Rani Mari, Anandhi \\ Eswaran
}

\begin{abstract}
Vetiveria zizanioides has been assigned for the extraction of phenolic acids and flavonoids for soluble, glycoside and wall-bound fractions. There was the largest number of phenolic acids and flavonoids in the methanolic extract that constitutes the cell wall-bound portion. Free radicals can induce biomolecules to oxidize, resulting in cell damage and countless illnesses. The present study investigates the role of enzymatic antioxidants, i.e. catalase, superoxide dismutase, glutathione peroxidase,glutathione reductase. Vitamin Eand C enzyme activity was nonenzymaticantioxidant action by spectrophotometric method. The enzymatic glutothi-one peroxidase antioxidant was found to be exampling than the rest while Vitamin $E$, notified found to be best activity ratherthan Vitamin C.The reversed highperformance liquid chromatographic techniquewas created and validated for the concurrent identification of free phenolic acids and flavonoids using a photodiode array detector with gradient elution.(Caffeicacid, Hydroxy benzoic acid, Rutin, Quercetin, Para- cumaric acid and Kaempferol) in the methanolic root extract of Vetiveria zizanioides.
\end{abstract}

Index Terms: Vetiveria Zizanioides, enzymatic antioxidants, Non-enzymatic antioxidants, HPLC analysis.

\section{INTRODUCTION}

Herbal drugs are currently available and are becoming incre asingly common on a daily basis. Herbal plants have effective components that are primarily used to prevent or cure diseases [1]. They may also have other characteristics and generally do so that they can be used as botanical pesticides, incenses, preservatives, herbal teas, organic dyes, herbal drinks, spices, oils, etc [2]. From the biological system, free radicals or ROS are produc ed which can alsoconduct the disease by destroying biomole cules. Phytochemicals are connected with the defense of human physical condition against the chronic degenerative

Revised Manuscript Received on September 25, 2019

Punithavathi Manogaran, Kavitha Rani Mari, Anandhi Eswaran, Ph.D Research Scholars, Department of Biochemistry, Periyar University, Salem

Dr. Suriyavathana Mthukrishnan, Associate Professor, Department of Biochemistry, Periyar University, Salem, India. Suriyabio14@gmail.com diseases which is also the bioactive substances of plants [3] Antioxidants assist us prevent cell damage caused by free ra dicals by inhibiting or slowing down the oxidizing cell resp onses [4].

Superoxide dismutases are a category of tightly associated e nzymes thatcatalyze the oxide and hydrogen peroxide conve rsion of the superoxide anion.Flavoprotein, glutathione red uctase, utilizes the reduction energy of the pentose phosphat e pathway (NADPH) to maintain the glutathione reservoir $i$ $\mathrm{n}$ a reduced state in the cell.In latest years, Vetiver grass ha $\mathrm{s}$ been commonly recognized for its efficacy in erosion and control of sediments, as well as being extremely capable of severe soil circumstances[5].

Highperformance liquid chromatography (or) Highpressure fluid chromatography (HPLC) is a specific form of matrix c hromatography widely used in biology and assessment to id entify, identify and quantify significant compounds[6].HPL $\mathrm{C}$ primarily uses a column holding the packaging content (s tationary phase), a pump moving the mobile phase(s) throu gh the column, and a detectorshowing the retention times of the molecules.Retention period differs based on therelations hips between the 15 static phase, the tested molecules and $\mathrm{t}$ he solvent(s) used. The sample to be evaluated is brought into the mobile phase flux in tiny quantity and is delayed by particular physical or chemical interactions with the stationary phase [7].

The quantity of retardation relies on the type of the analysis and the static and mobilePhase structure. The moment when a particular analyte elutes (originates from the end of the column) is termed the retention time [8]. The configuration of the mobile phase, known as gradient $\mathrm{e}$ lution, was performed during the analysis [9]. As a result of the analyte affinity for the present mobile phase, the gradient divides the analyte mixtures [10]. Depending on the design of the stationary phase and the analyte, the selection of solvents, additives and gradient.

\section{MATERIAL AND MATERIALS}

Collection of Plant Sample

From Kolli Hills, Namakkal District, Tamilnadu, India, the root of Vetiveria zizanioides was obtained.

Preparation of Plant Extract

To achieve a homogeneous sample, the roots were crashed with pestle and mortar. Crude samples were ready in $400 \mathrm{ml}$ of distilled water individually by grinding $100 \mathrm{~g}$ of each plant. 


\section{HPLC Characterization and Assessment of Antioxidant Status of Vetiveria Zizanioides Roots}

By blending for 15 minutes, the crushed extracts were Carefully homogenized and centrifuged for $15 \mathrm{~min}$ at 6000 RPM. The supernatant was filtered through sieve, discarding the sediment. After they were preserved at $-10^{\circ} \mathrm{C}$.

\section{Vetiveria zizanioides root extract enzymatic antioxidant activity}

Catalase activity was determined by the operation, the process of determining the Superoxide dismutase function, Glutathione Peroxidase was analyzed technique and Glutathione reductase activation technique.

\section{Non- Enzymatic Antioxidants OfVetiveriaZizanioidesroot extract}

Ascorbic acid (VitaminC) concentrations were evaluated us ing technique. And the technique of estimating the concentrations of vitamin-E.

\section{Characterization OfVetiveriaZizanioides Root Extract by HPLC analysis}

High-performance liquid chromatography (HPLC) using th e Sachan procedure.

\section{RESULTS AND DISCUSSION}

Enzymatic antioxidant activity of Vetiveria zizanioides root extract

In ordinary and pathological cell metabolism, free radicals comprise one or more

unpaired electrons, with this free radical, reactive oxygen $\mathrm{sp}$ ecies (ROS) respond readily to

become radical themselves.In living organisms, they are for med by various techniques, such

as aerobic respiration that stimulates macrophages and poly morphic nuclear glycoside and

peroxisomes which are a normalbyproduct the metabolism of our body.However, if there is an accumulation of biomole cule such as lipids, protein, DNA, RNA resulting cell or tiss ue injury, the counteroxidant stress our body acts naturally $r$ elies on the manner in which antioxidant enzymes are synth esized.The antioxidant enzymes are glutathione reductase, glutathione peroxidase, superoxide dismutase, and catalase thatprevents celldamage and injury by offering full naturalp rotectionagainst ROS. The status of antioxidant enzymes present in Vetiveriazizanioidesroot is depicted in the Table-1 and Figure-1,the content of enzymatic antioxidantsdetermined using standards protocols reveals that Vetiveriazizanioidesroot exhibited high GPX (0.84) activity followed by SOD (0.709) besides this the level of GR (0.42) and CAT (0.22) was noted to be less.

The quenching of the singlet oxygen (Superoxide) which is likely to be the main causative and drastic free radial species which will adhere to be cell membrane there by causing serve oxidative damage to the cell and tissue in the naturals resources namely Vetiveriazizanioidesroot possess very good store of effective antioxidant enzymes namely superoxide dismutase and glutathione peroxidase the quantification of this antioxidant enzymes from Vetiveriazizanioides root revealed an eye opening to the research on natural products indulged with to explore the valuable bioactive and biochemical constituents in it which has been experimental and exhibited by this systematic quantification analysis in Vetiveriazizanioides root

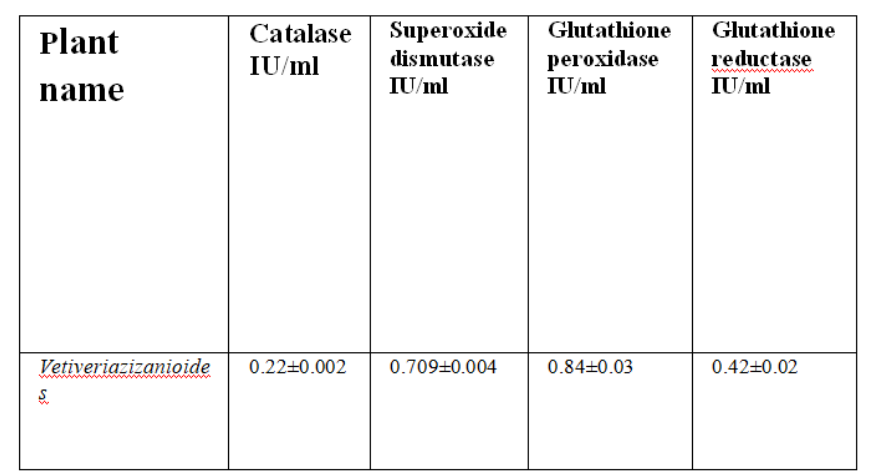

Table1Enzymatic antioxidant activity of Vetiveriazizanioides root extract

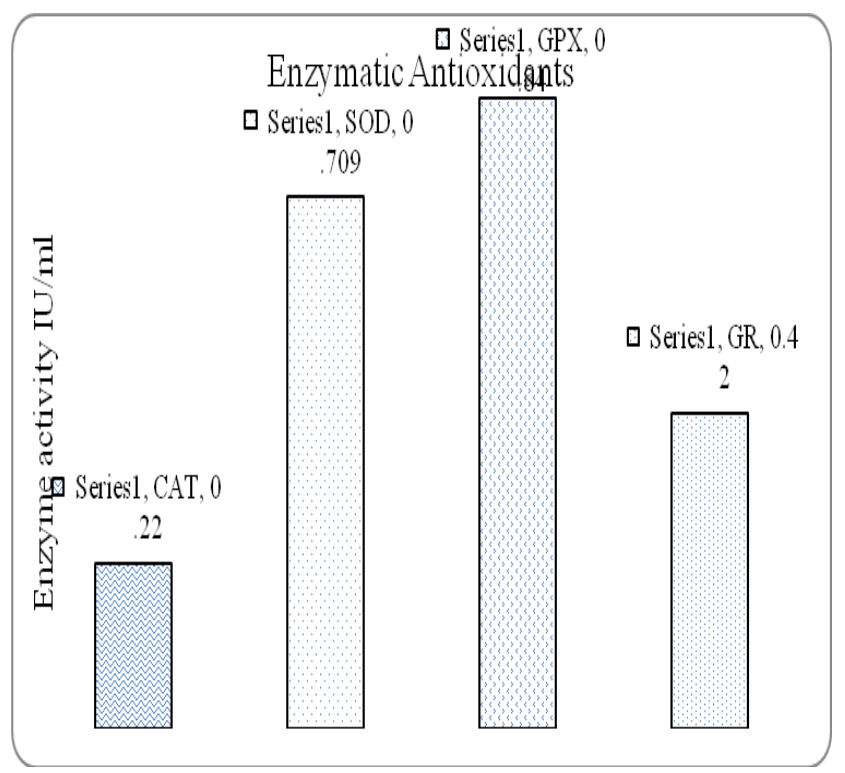

Fig.1Vetiveria zizanioides root extract's enzymatic antioxidant activity

Catalase is an enzyme which is limited in this effectiveness to metabolise $\mathrm{H}_{2} \mathrm{O}_{2}$ to $\mathrm{H}_{2} \mathrm{O}$ by its relatively poor affinity for $\mathrm{H}_{2} \mathrm{O}_{2}$ and its subcellular location, in peroxisomes is susceptible to photo inactivation and degradation. Superoxide dismutase (SODs) are a category of enzymes that catalyse the oxide and hydrogen peroxide conversion of the superoxide anion. Glutathione peroxidases (Gpxs) are omnipresent proteins that catalyse glutathione to reduce hydrogen peroxides and organic hydrogen peroxides. Desmodiumgangeticum 164.04 $\pm 1.9,789.7 \pm 2.19,166 \pm 1.87$ $\mu \mathrm{g} / \mathrm{mg}$ levels of the enzymatic antioxidant namely glutathi one peroxidase, superoxide dismutase and catalase. 
Non- Enzymatic Antioxidants of VetiveriaZizanioidesRoots

Vetiveria zizanioides root's nonenzymatic antioxidants, incl uding vitamin $\mathrm{E}$ and vitamin $\mathrm{C}$,were shown in table 2 and figure.2. The essential and nutritional important of minerals and vitamins which has been continuously emphasized in the field of nutrition and growth development mechanism in living system. The important of this vital amines = vitamins $\mathrm{C}, \mathrm{E}$ which has been known to have very crucial and health beneficial effects on mankind for the healthy life existence. The presence of the non-enzymatic antioxidants not only serving as a nutritional component parallelly but it acts as an effective non enzymatic antioxidant as its other parts vitamin. The level of vitamin $\mathrm{E}$ (Tocopherol) is noted to be higher than vitamin-C which has been determined from the Vetiveriazizanioides roots.

\begin{tabular}{|l|l|l|l|l|l|l|}
\hline \multicolumn{7}{|l|}{ Detector $340(\mathbf{n m})$} \\
\hline Peak\# & Ret. Time & Area & Height & Area \% & Height \% & Name \\
\hline 1 & 3.447 & 7699462 & 52430 & 83.091 & 76.691 & Caffeic acid \\
\hline 2 & 7.140 & 1262978 & 12544 & 13.630 & 18.348 & Hydroxy benzoic acid \\
\hline 3 & 10.096 & 297867 & 3056 & 3.215 & 4.470 & Rutin \\
\hline 4 & 14.252 & 1165 & 56 & 0.013 & 0.082 & Quercetin \\
\hline 5 & 15.139 & 1071 & 136 & 0.012 & 0.200 & Para- cumaric acid \\
\hline Total & & 9266255 & 68366 & 100.000 & 100.000 & \\
\hline
\end{tabular}

Table-2 Non- Enzymatic antioxidant activity of Vetiveriazizanioidesroot extract

This is determined from the Vetiveriazizanioides root the presence of these importance non enzymatic along with high content of enzymatic antioxidant in the same sources namely Vetiveriazizanioides root which is predicted to be an a valuable natural sources of antioxidant store. Revealed that by modifying mechanisms from mitosis and cell elongation to senescence and death, non-enzymatic antioxidants can influence plant growth anddevelopment.

Similarly, abiotic stressrelated gene expression changes the level of nonenzymatic antioxidants including vitamin $\mathrm{C}$, vit amin $\mathrm{E}$, andreduced glutathione28.24 $\pm 0.9,69.54 \pm 1.53,2$. $98 \pm 0.80$ in Desmodium gangeticum.

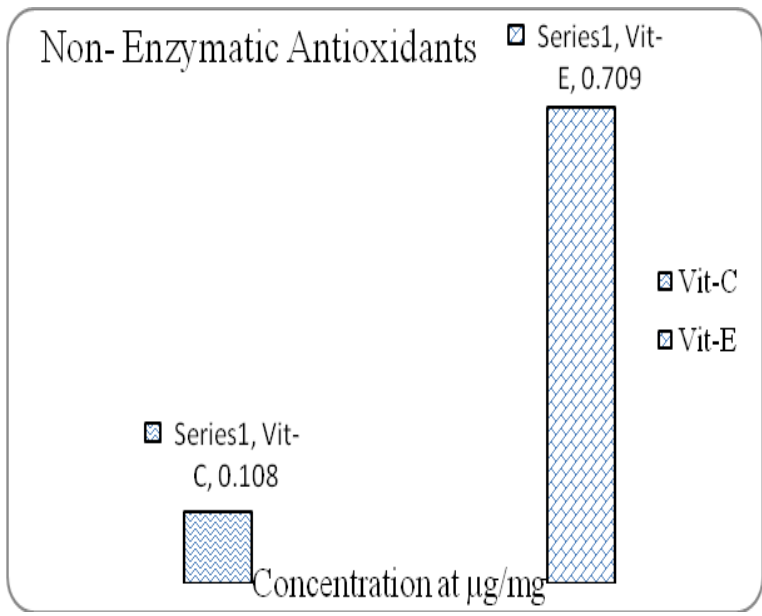

Fig. 2 Non- Enzymatic antioxidant activity of Vetiveriazizanioides root extract

Characterization of Vetiveriazizanioides Root Extract by HPLC analysis

HPLC chromatogram of phenolic compounds from methanolic root extract of Vetiveriazizanioideswas presented in fig- 3 and the data were presented in table- 3.In the methanolic root extract of Vetiveria zizanioides, hydroxy benzoic acid, coffeic acid, paracuemaric acid, quercetin and rutin were recognized by combining their Retention Time (RT) and UV spectrum of authentic standards evaluated under the same circumstances, whereas the relative information from their corresponding calibration graphs weredetermined.In retention times of 3,447 minutes and 7,1 40 minutes respectively, coffeic acid and hydroxyl benzoic acid were recognized.No definite elevations were noted link ed toparacuemaric acid,quercetin, andrutin he levels of thes e elements in the Vetiveria zizanioides methanolic root extr act.

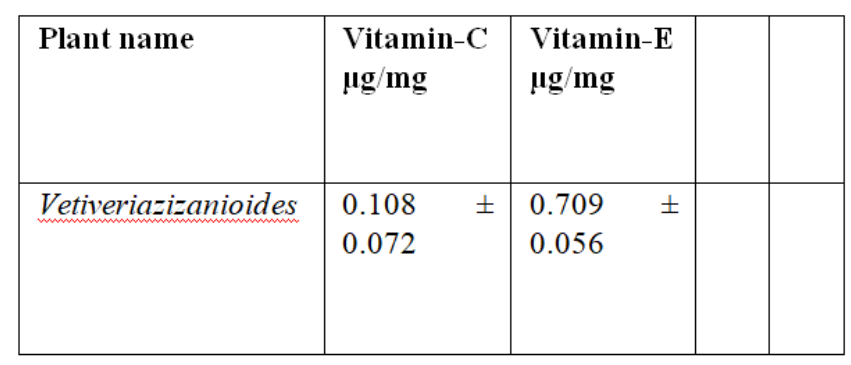

Table-3 HPLC Analysis of Phenolic compounds From Methanolic root extract of Vetiveria Zizanioides ॥V

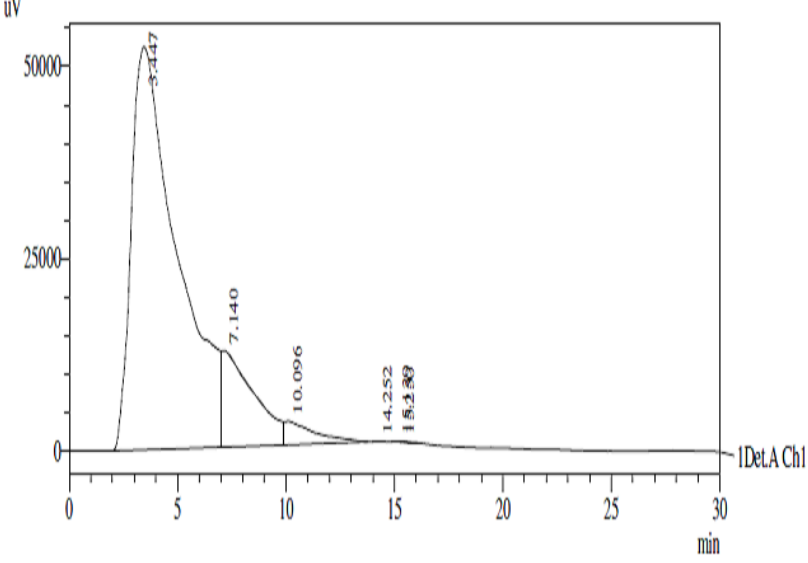

Fig. 3 HPLC Analysis of phenols From methanolic root

\section{CONCLUSION}

The expedition to research of plant based natural products has been constantly mapped and localization of these bioactive compounds namely phytochemicals of Vetiveria zizanioides roots has been explored from kollihills, Namakkal (Dt). The systematic screening and 
quantification provided a very valuable and interesting facts that Vetiveria zizanioides roots upholds a wide range of Antioxidant enzymes the sources opted has comprehensively validates that Vetiveria zizanioides roots will certainly act as an effective and therapeutic natural resource with detailed characterization and in vivo experimental studies are wanted in order to expose this valid enriched Vetiveria zizanioides roots to the society.

\section{ACKNOWLEDGEMENTS}

The authors Punithavathi Manogaran and Suriyavathana Muthukrishnan are overwhelmed to the Periyar University for providing financially support (URF) to carry this work.

\section{REFERENCES}

1. Chomchalow N, Production of Medicinal and Aromatic Plants in Southeast Asia. AU Journal of technology. 2000 Oct 4(1): 84-94.

2. Dipjyoti C. HPLC quantification of phenolic acids from Vetiveria zizanioides (L.) Nash and its antioxidant and antimicrobial activity. Journal of pharmaceutics. 2013 Mar 11;2013.

3. Edziri HL, Smach MA, Ammar S, Mahjoub MA, Mighri Z, Aouni M, Mastouri M. Antioxidant, antibacterial, and antiviral effects of Lactucasativa extracts. Industrial Crops and Products. $2011 \mathrm{Ju}$ $1 ; 34(1): 1182-5$.

4. Florence AR, Joselin J, Brintha TS, Sukumaran S, Jeeva S. Preliminary phytochemical studies of select members of the family Annonaceae for bioactive constituents. BiosciDiscov. 2014;5(1):85-96.

5. Fukumoto LR, Mazza G. Assessing antioxidant and prooxidant activities of phenolic compounds. Journal of agricultural and food chemistry. 2000 Aug 21;48(8):3597-604.

6. Gülçin İ, Elmastas M, Aboul-Enein HY. Antioxidant activity of clove oil-A powerful antioxidant source. Arabian Journal of chemistry. 2012 Oct 1;5(4):489-99.

7. Martin M, Guiochon G. Effects of high pressure in liquid chromatography. Journal of Chromatography A. 2005 Oct 7;1090 (1-2):16-38.

8. Sutapun W, Suppakarn N, Ruksakulpiwat Y. Study of Characteristic of VetiverFiberBefore and after Alkaline Treatment. In Advanced Materials Research 2010 Aug 11;123:1191-1194.

9. Sachan A, Ghosh S, Mitra A. An efficient isocratic separation of hydroxycinnamates and their corresponding benzoates from microbial and plant sources by HPLC. Biotechnology and applied biochemistry. 2004 Oct;40(2):197-200.

10. Xiang Y, Liu Y, Lee ML. Ultrahigh pressure liquid chromatography using elevated temperature. Journal of Chromatography A. $2006 \mathrm{Feb}$ 3;1104 (1-2):198-202.

11. Ganesan Vijaiyan Siva. Spectrochim. Acta, Part A., 2014, vol. 127, pp. 61-66. 